\title{
Charge Hall effect driven by spin-dependent chemical potential gradients and Onsager relations in mesoscopic systems
}

\author{
E. M. Hankiewicz, ${ }^{1}$ Jian Li, ${ }^{2}$ Tomas Jungwirth, ${ }^{3,4,5}$ Qian Niu, ${ }^{5}$ Shun-Qing Shen, ${ }^{2}$ and Jairo Sinova ${ }^{1}$ \\ ${ }^{1}$ Department of Physics, Texas A\&M University, College Station, Texas 77843-4242, USA \\ ${ }^{2}$ Department of Physics, The University of Hong-Kong, Pukfulam Road, Hong-Kong, China \\ ${ }^{3}$ Institute of Physics ASCR, Cukrovarnická 10, 16253 Praha 6, Czech Republic \\ ${ }^{4}$ School of Physics and Astronomy, University of Nottingham, Nottingham NG7 2RD, United Kingdom \\ ${ }^{5}$ Department of Physics, University of Texas, Austin, Texas 78712-0264, USA
}

(Received 25 May 2005; published 7 October 2005)

\begin{abstract}
We study theoretically the spin-Hall effect as well as its reciprocal phenomenon (a transverse charge current driven by a spin-dependent chemical potential gradient) in electron and hole finite size mesoscopic systems. The Landauer-Buttiker-Keldysh formalism is used to model samples with mobilities and Rashba coupling strengths which are experimentally accessible and to demonstrate the appearance of measurable charge currents induced by the spin-dependent chemical potential gradient in the reciprocal spin-Hall effect. We also demonstrate that within the mesoscopic coherent transport regime the Onsager relations are fulfilled for the disorder averaged conductances for electron and hole mesoscopic systems.
\end{abstract}

DOI: 10.1103/PhysRevB.72.155305 PACS number(s): 73.23. - b, 72.25.Dc, 72.25.Hg, 85.75. $-\mathrm{d}$

\section{INTRODUCTION}

In the very active field of semiconductor based spintronics the control of spin can be achieved by the manipulation of the strength of spin-orbit (SO) interactions in paramagnetic systems. Within this context, the newly proposed intrinsic spin-Hall effect (SHE) in p-doped semiconductors by $\mathrm{Mu}-$ rakami et al. ${ }^{1}$ and in a two-dimensional electron system (2DES) by Sinova et al. ${ }^{2}$ offers new possibilities for spin current manipulation and generation in high mobility paramagnetic semiconductor systems. In contrast to the earlier proposed extrinsic spin-Hall effect, ${ }^{3-5}$ which is associated with scattering from impurities, the intrinsic spin-Hall arises purely from host semiconductor band structure and represents a spin-current response generated perpendicular to the driving electric field.

Recently, the spin Hall effect was experimentally observed by Kato et al. ${ }^{6}$ in $n$-doped GaAs using the Kerr effect and by Wunderlich et $a .^{7}$ in the $p$ - $n$ junction light-emitting diodes based on two-dimensional hole gas (2DHG) (Al,Ga)As. Although the experiment by Wunderlich et al. seems to be in the regime where the intrinsic effect in $2 \mathrm{DHG}$ is dominant, the main theoretical focus has been concentrated so far on 2DEG with Rashba SO interactions, where Rashba term is linear with $k^{2,8-21}$ The influence of disorder on infinite 2DEG is still unclear (for a recent review see Ref. $21)$. For $\delta$-function impurities the analytical calculations of vertex corrections in the ladder approximation seem to cancel the intrinsic spin-Hall effect in a weak scattering regime. ${ }^{12,14}$ However, these calculations have been challenged recently. ${ }^{20}$ Further, the numerical calculations for 2DEG based on Kubo formula using continuum model in momentum space ${ }^{13}$ and discrete model in the real space $^{22}$ show finite spin-conductivity in a weak scattering regime which goes to zero in the thermodynamic limit. ${ }^{22,23}$ In contrast, for the infinite $2 \mathrm{DHG}$ the vertex corrections vanish. ${ }^{24,25}$ Further the numerical calculations for $2 \mathrm{DHG}$ based on Kubo formula using continuum model in momentum space show the finite value of SHE in a weak scattering regime which goes to constant in the thermodynamic limit. ${ }^{23}$

The calculations within the Landauer-Buttiker (LB) formalism on finite size systems ${ }^{17-19,26}$ model a sample of micro/nanosize attached to contacts. The calculations on electron mesoscopic systems show that spin Hall conductance is a fraction of $e / 8 \pi$ in a weak scattering regime. ${ }^{17-19}$ Moreover, a mesoscopic spin-Hall conductance is robust against the disorder. ${ }^{17-19}$ Very recently $\mathrm{Wu}$ and Zhou considered the Luttinger model, ${ }^{27}$ showing as expected that SHE can be much larger in hole systems in comparison with the electron ones. Although the experimental measurement by Wunderlich et al. ${ }^{7}$ concern 2DHG systems with broken inversion symmetry, the pure cubic Rashba term was not considered in detail within the LB formalism so far.

The observation of spin-Hall effect through transport measurements is one of the urgent experimental challenges facing this spin-transport physics. Recently, an H-probe structure has been proposed to measure the effect where the spin-Hall effect could be measured indirectly by detecting charge voltages induced by the reciprocal spin-Hall effect (RSHE). ${ }^{19}$ This RSHE, where transverse charge current is driven by spin dependent chemical potential, was proposed in a context of extrinsic spin-Hall effect by Hirsch ${ }^{4}$ and formulated in a semiclassical approach by Zhang and Niu. ${ }^{28}$ Also, the Onsager relation between the spin-Hall conductivity and reciprocal charge-Hall conductivity was established within a wave packet model through a redefinition of the spin-current including spin-torque terms in the bulk. ${ }^{28} \mathrm{We}$ show here that within the mesoscopic regime, and more specifically within the Landauer-Buttiker-Keldysh formalism, the Onsager relations are satisfied within the models studied for the disorder averaged conductances. Because the conductances are formulated with respect to the leads which have no spin-orbit coupling, it is not necessary nor consequential to introduce the spin current redefinition in our problem. ${ }^{28}$ 
In this paper we compare the magnitude of the SHE as well as the RSHE in finite electron and hole mesoscopic systems within the LB formalism. We show that the conductances associated with both effects are significantly larger in the hole systems. Furthermore, we analyze the possible experimental setup to measure the RSHE. We show that the charge current driven by a spin-dependent chemical potential gradient is on the order of hundred nano-ampers for typical voltages in hole systems and should be experimentally measurable.

\section{MODEL HAMILTONIAN FOR HOLE SYSTEM AND LB TREATMENT OF THE SPIN-HALL EFFECT AND ITS RECIPROCAL CORRESPONDENT}

The observation of the spin-Hall effect and its reciprocal phenomenon in transport is the next experimental challenge in the subfield of spintronics using spin-orbit interactions to manipulate the spin.

The continuum effective mass model for $2 \mathrm{DHG}$ in a narrow inversion asymmetrical well is given by: ${ }^{29} \hat{H}=\hat{p}^{2} / 2 m^{*}$ $+i\left(\lambda / 2 \hbar^{3}\right)\left(p_{-}^{3} \hat{\sigma}_{+}-p_{+}^{3} \hat{\sigma}_{-}\right)+H_{d i s}$, where $H_{d i s}$ describes disorder. We use the tight-binding approximation ${ }^{30}$ to model the disordered conductor within the LB formalism. Within this approximation the continuum effective mass envelope function Hamiltonian becomes:

$$
\begin{aligned}
H= & \sum_{j, \sigma} \epsilon_{j} c_{j, \sigma}^{\dagger} c_{j, \sigma}-t \sum_{j, \boldsymbol{\delta}, \sigma} c_{j+\boldsymbol{\delta}, \sigma}^{\dagger} c_{j, \sigma}+a_{0}^{2} t_{S O-k^{3}}\left[\sum_{j} c_{j, \uparrow}^{\dagger} c_{j+2 a_{x} \downarrow}\right. \\
& -c_{j, \uparrow}^{\dagger} c_{j-2 a_{x} \downarrow}+i \sum_{j} c_{j, \uparrow}^{\dagger} c_{j+2 a_{y} \downarrow}-c_{j, \uparrow}^{\dagger} c_{j-2 a_{y}, \downarrow} \\
& +3(1-i) \sum_{j} c_{j, \uparrow}^{\dagger} c_{j-a_{x}+a_{y}, \downarrow}-c_{j, \uparrow}^{\dagger} c_{j+a_{x}-a_{y}, \downarrow} \\
& +3(1+i) \sum_{j} c_{j, \uparrow}^{\dagger} c_{j-a_{x}-a_{y}, \downarrow}-c_{j, \uparrow}^{\dagger} c_{j+a_{x}+a_{y}, \downarrow}+4 i \sum_{j} c_{j, \uparrow}^{\dagger} c_{j+a_{y}, \downarrow} \\
& \left.-c_{j, \uparrow}^{\dagger} c_{j-a_{y}, \downarrow}+4 \sum_{j} c_{j, \uparrow}^{\dagger} c_{j+a_{x}, \downarrow}-c_{j, \uparrow}^{\dagger} c_{j-a_{x}, \downarrow}+\text { H.c. }\right],
\end{aligned}
$$

where $t=\hbar^{2} / 2 m^{*} a_{0}^{2}$ and $t_{S O-k^{3}}=-\lambda / 2 a_{0}^{3}, a_{0}$ is the mesh lattice spacing, and $\boldsymbol{\delta}= \pm a_{0} \hat{x}, \pm a_{0} \hat{y}$. The first term represents a quenched disorder potential and disorder is introduced by randomly selecting the on-site energy $\epsilon_{j}$ in the range $[-\mathrm{W} / 2, \mathrm{~W} / 2]$. The continuum effective mass model for 2DES and its tight-binding correspondent can be found elsewhere. ${ }^{18,19}$

Within the leads the SO coupling is zero and therefore each lead should be considered as having two independent spin-channels. Moreover, in leads without the SO coupling, the spin current is measured in a medium where spin is conserved removing the ambiguity of spin-current definition. For SHE, leads constitute reservoirs of electrons at chemical potential $\mu_{1}, \ldots, \mu_{N}$, where $N$ is the number of leads that we consider to be four [see Fig. 1(b)]. For RSHE, the chemical potential is spin dependent in leads 1 and 2 allowing the

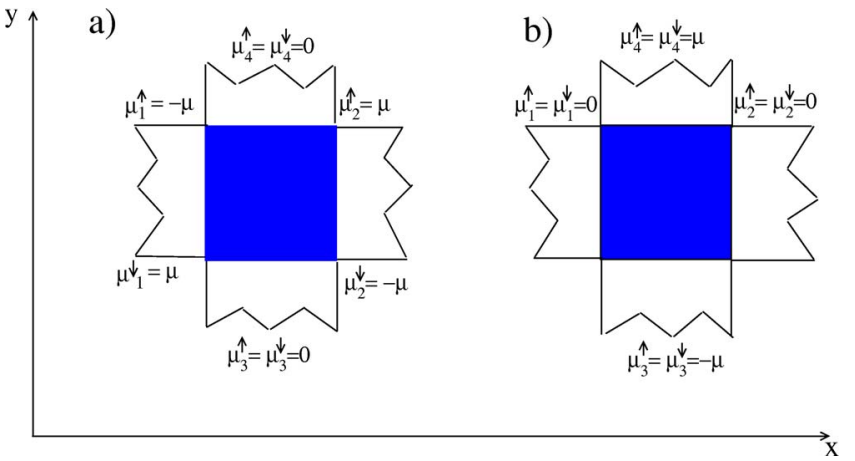

FIG. 1. (Color online) The schematic picture of setup to measure (a) charge Hall effect driven by spin dependent chemical potential and (b) spin-Hall effect.

generation of spin-force in the $x$ direction [see Fig. 1(a)]. In the low temperature limit $k_{B} T \ll E_{F}$ and for low bias-voltage, the particle current going through a particular channel is given within the LB formalism by $I_{p, \sigma}$ $=(e / h) \sum_{q \sigma^{\prime}} T_{p, \sigma ; q, \sigma^{\prime}}\left[V_{p}-V_{q}\right]$, where $p$ labels the lead and $T_{p, \sigma ; q, \sigma^{\prime}}$ is the transmission coefficient at the Fermi energy $E_{F}$ between the $(p, \sigma)$ channel and the $\left(q, \sigma^{\prime}\right)$ channel. This transmission coefficient is obtained by $T_{p, \sigma ; q, \sigma^{\prime}}$ $=\operatorname{Tr}\left[\Gamma_{p, \sigma} G^{R} \Gamma_{q, \sigma^{\prime}} G^{A}\right]$ where $\Gamma_{p, \sigma}$ is given by $\Gamma_{p, \sigma}(i, j)$ $=i\left[\Sigma_{p, \sigma}^{R}(i, j)-\Sigma_{p, \sigma}^{A}(i, j)\right]$. The retarded and advanced Green's function of the sample $G^{R / A}$ with the leads taken into account through the self energy $\Sigma_{p, \sigma}^{R / A}(i, j)$ has a form $G^{R / A}(i, j)$ $=\left[E \delta_{i, j}-H_{i, j}-\Sigma_{p, \sigma} \Sigma_{p, \sigma}^{R / A}(i, j)\right]^{-1}$. Here the position representation of the matrices $\Gamma_{p, \sigma}, G^{R}, H_{i, j}$, and $\Sigma^{R}$ are in the subspace of the sample. Within the above formalism the spin current through each channel is given by $I_{p, \sigma}^{s}$ $=(e / 4 \pi) \sum_{q \sigma^{\prime}} T_{p, \sigma ; q, \sigma^{\prime}}\left[V_{p, \sigma^{-}}-V_{q, \sigma^{\prime}}\right]$. The spin force driven charge-Hall conductance, $G_{C S}^{y x}$, is defined as the ratio of charge current in the $y$ direction induced by the spindependent chemical potential along the $x$ axis to this spindependent chemical potential difference [see Fig. 1(a)].

$$
G_{C S}^{y x}=\frac{\left(I_{3 \uparrow}^{S}+I_{3 \downarrow}^{s}\right)}{V_{2}^{\uparrow}-V_{1}^{\uparrow}},
$$

where $V_{i}^{\uparrow}=\mu_{i}^{\uparrow} / e$. The spin-Hall conductance, $G_{S C}^{x y}$, is defined as the ratio of spin-current in the $x$ direction induced by charge voltage difference in $y$ direction to this voltage difference

$$
G_{S C}^{x y}=\frac{\left(I_{1 \uparrow}^{s}-I_{1 \downarrow}^{s}\right)}{V_{4}-V_{3}},
$$

where $V_{i}=\mu_{i} / e$, and the labels are indicated in Fig. 1(b). $G_{S C}^{y x}$ and $G_{C S}^{x y}$ are defined by analogy. We set the absolute value of voltage for spin and spin force driven charge-Hall effects as $V=\mu / e=2.5 \mathrm{mV}$.

\section{RESULTS AND DISCUSSION}

In order to address the key issue of experimental observation of spin force driven charge-Hall effect as well as to establish the Onsager relation between spin-Hall effect and it 
(a)

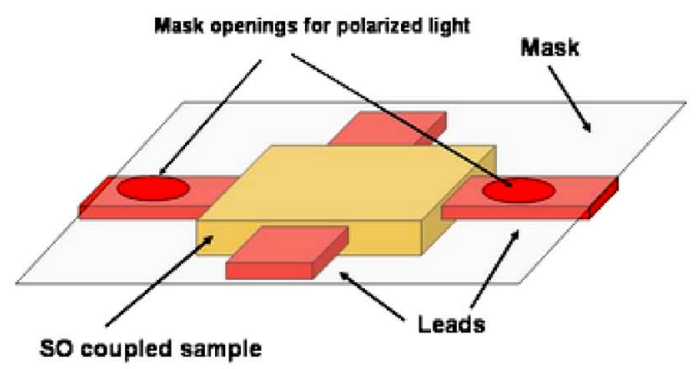

(b)

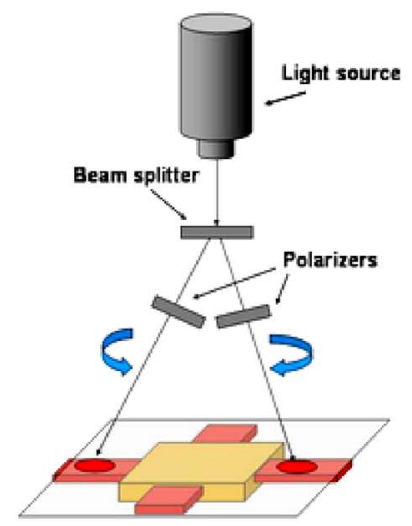

FIG. 2. (Color online) Proposal of experimental setup to measure the reciprocal spin-Hall effect. (a) Mask (light-gray) covers a sample and leads except for two holes in the longitudinal leads where circularly polarized light shines. (b) Full schematics of the experimental setup. reciprocal correspondent we choose realistic parameters for our calculations which model currently attainable systems. We consider an effective mass of $m^{*}=0.05 m_{e}$ for electron systems and $m^{*}=0.5 m_{e}$ for hole ones. The disorder strength $W=0.09 \mathrm{meV}$ corresponds to the mobility of $250000 \mathrm{~cm}^{2} / \mathrm{Vs}$, which is typical for a semiconductor like (In,Ga)As. We take the Rashba parameter $\lambda$ in the range from 0 to $100 \mathrm{meV} \mathrm{nm}$, easily obtained in experiments, ${ }^{31,32}$ and we choose the electron concentration $n_{2 D}$ in a range between $3 \times 10^{11} \mathrm{~cm}^{-2}$ and $1.3 \times 10^{12} \mathrm{~cm}^{-2}$. The Fermi energy is obtained from the chosen carrier concentration assuming an infinite two-dimensional (2D) gas. Parameters considered here correspond to $t_{s o} \approx 0-0.2 t, \quad E_{F} / W$ $\approx 500-800$ in other theoretical studies ${ }^{17,18}$ with small variation due to mesh scaling as physical system size and effective masses for hole and electron systems are kept constant. The Fermi energy is close to the bottom of the band and $E_{F}$ changes from $-3.9 t$ to $-3.5 t$ in units of Refs. 17 and 18 dependent on electron concentration.

In the detection of spin force driven charge-Hall effect the first task is to generate a spin force which can be realized by spin dependent chemical potential in the leads. The ferromagnetic leads are not the good candidates because although magnetization exists in ferromagnetic leads, the chemical po-

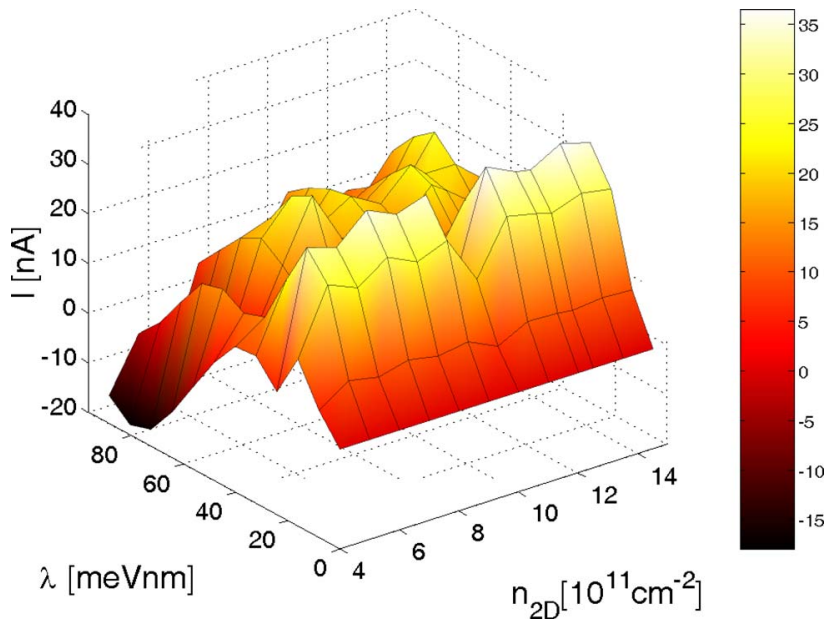

FIG. 3. (Color online) The spin-force driven charge current as a function of electron concentration and Rashba coupling, $\lambda$, for mesoscopic square sample $100 \mathrm{~nm}$ by $100 \mathrm{~nm}$ and $\mu$ $=250000 \mathrm{~cm}^{2} / \mathrm{Vs}$. tential is the same for both spin directions. Here, we propose the optical method of spin-dependent chemical potential generation by shining the beam of circularly polarized light on the leads (see Fig. 2). The right-circularly polarized beam shines on the right lead [lead 2 in Fig. 1(a)] and the left circularly polarized beam on the left one [lead 1 in Fig. 1(a)]. The sample as well as the transverse leads should be covered by mask, preventing the light absorption anywhere except the small part of longitudinal leads as shown in Fig. 2(a). Choosing semiconductor leads for this setup, e.g., GaAs, will cause the opposite spin polarizations in left and right leads through optical selection rules. Using the beam splitter should produce the same light intensity in each leads providing simultaneously the spin-dependent chemical potential between leads 1 and 2 and the total charge current across a sample equal zero. Having produced the spin-dependent chemical potential in the leads, we perform calculations using nonequilibrium Green function method presented in previous section. Figures 3 and 4 present the charge current $I_{3}$ $=I_{3 \uparrow}^{S}+I_{3 \downarrow}^{S}$ [see sample configuration Fig. 1(a)] as a function of SO coupling $\lambda$ and electron or hole concentrations, respectively. The charge current for electron systems show oscillations with respect to the electron density and SO coupling.

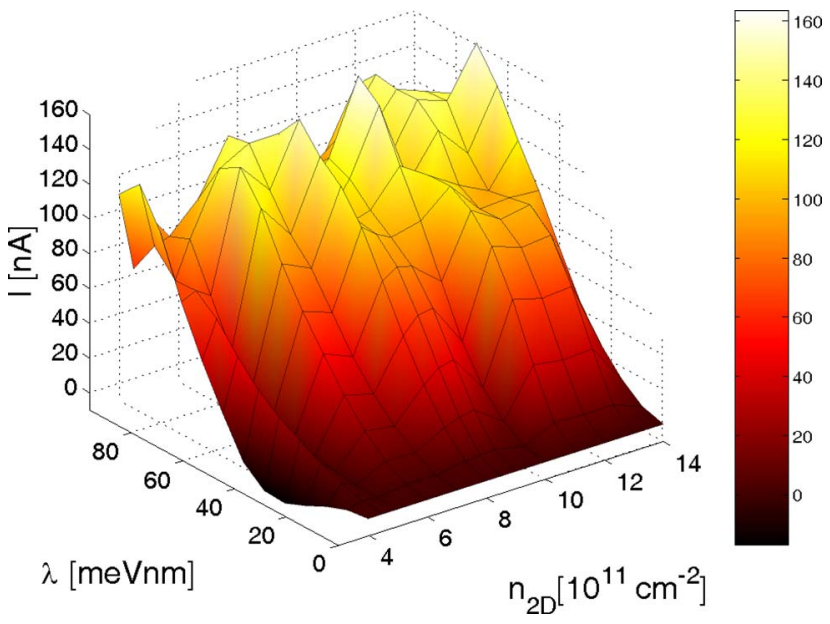

FIG. 4. (Color online) The spin-force driven charge current as a function of hole concentration and Rashba coupling, $\lambda$, for mesoscopic square sample $100 \mathrm{~nm}$ by $100 \mathrm{~nm}$ and $\mu=250000 \mathrm{~cm}^{2} / \mathrm{Vs}$. 


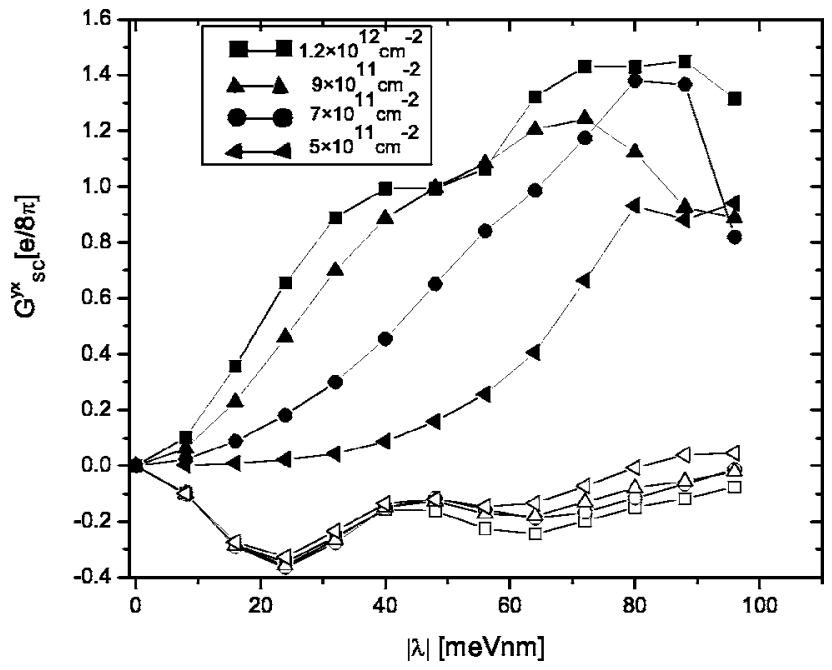

FIG. 5. The spin-Hall conductance as a function of SO coupling for $100 \mathrm{~nm}$ by $100 \mathrm{~nm}$ square system for different concentration of holes (close symbols) and electrons (open symbols).

The period of current oscillations depends on the system size, however, its maximal value seems to be around $40 \mathrm{nA}$ for systems sizes achievable in our calculations. For hole mesoscopic systems (see Fig. 4), the charge current also oscillates with $\lambda$ and for system sizes on the order of 100 nanometeres is on the order of hundred nanoampers in a wide range of densities starting from $6 \times 10^{11} \mathrm{~cm}^{-2}$. Hence the charge current is much larger in hole systems and shall be detectable in experiments.

Figure 5 presents the spin-Hall conductance $G_{S C}^{y x}$ as a function of $\lambda$ for electron and hole systems. One can see that spin-Hall conductances (Fig. 5) and spin-force driven charge conductances (see Figs. 3 and 4) behave similarly. Spin-Hall conductances for electron systems oscillate with SO coupling and have values of the fraction of $e / 8 \pi$ in agreement with results presented in Refs. 17-19. Similarly behave the spinHall conductances for hole systems. However, $G_{S C}^{y x}$ are several times larger for hole systems in comparison with electron ones, which is associated with much larger effective mass in a case of hole systems. Let us emphasize that for electron and hole systems with the same effective masses the spin-Hall effect can be larger for electron systems. Moreover, in mesoscopic systems where Fermi energy as well as multichannel effects are important the straightforward renormalization of effective mass of electron and hole systems to compare the spin-Hall conductance suggested by Ref. 27 does not have to be correct. Our calculations in mesoscopic systems are in agreement with the linear response Kubo calculation for 2DEG and 2DHG which show that spinconductance is much more larger for hole systems. ${ }^{2,29}$ The sign of spin-Hall and charge Hall conductances in mesoscopic systems depends on the Fermi energy.

The Onsager relations express the symmetry between the transport coefficients describing reciprocal processes in systems with a linear dependence of response to the driving forces. Within the models studied, considering disorder averaged conductances, the relations can be derived by utilizing the time reversal symmetry and the inversion symmetry

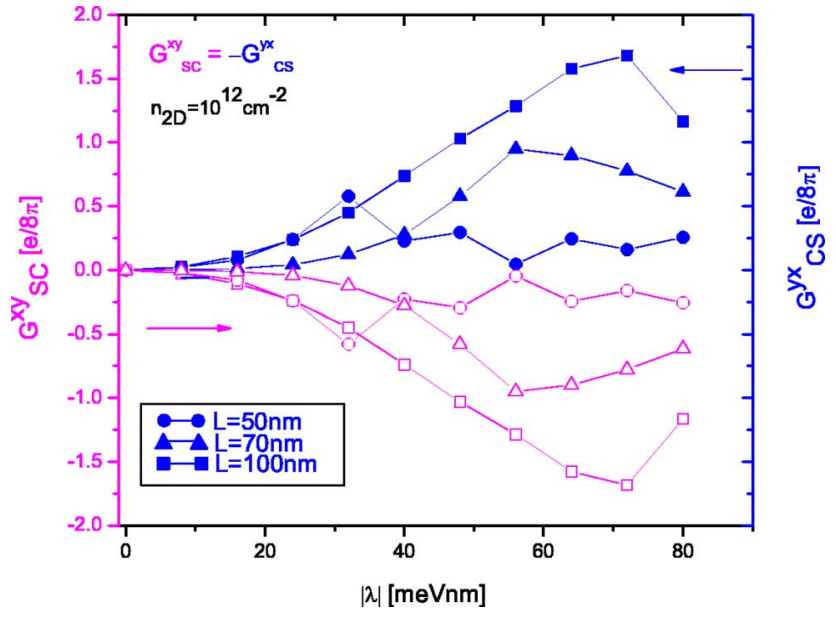

FIG. 6. (Color online) Onsager relation between the spin-Hall conductance $G_{S C}^{x y}$ (open symbols) and spin-force driven charge-Hall conductance $G_{C S}^{y x}$ (close symbols) for different $\mathrm{L}$ by $\mathrm{L}$ hole systems.

on the $x-y$ plane which relate $T_{\sigma n ; \sigma m}=T_{\bar{\sigma} m ; \bar{\alpha} n}$ and $T_{\sigma n ; \alpha m}$ $=T_{\sigma m ; \alpha n}$, respectively, where $\sigma, \alpha$ and $n, m$ are the spin and lead labels and a bar represents the opposite direction. Within the SHE $V_{1}^{\sigma}=V_{2}^{\sigma}=0$ and $V_{3}^{\sigma}=-V_{4}^{\sigma}=V_{0} / 2$ following the labels of Fig. 1. Within the RSHE $V_{3}^{\sigma}=V_{4}^{\sigma}=0$ and $V_{1}^{\sigma}=V_{2}^{\bar{\sigma}}$ $=s(\sigma) V_{0} / 2$ where $s(\uparrow)=+1$ and $s(\downarrow)=-1$. Within the LB formalism and these boundary conditions we obtain for the spin-current associated with the SHE:

$$
I_{1}^{\text {spin }} \equiv I_{1}^{\uparrow}-I_{1}^{\downarrow}=V_{0} \sum_{\sigma, \alpha} s(\sigma)\left(T_{\sigma 1 ; \alpha 3}-T_{\sigma 1 ; \alpha 4}\right) / 2,
$$

and for the charge current associated with the RSHE:

$$
I_{3}^{c} \equiv I_{3}^{\uparrow}+I_{3}^{\downarrow}=V_{0} \sum_{\sigma, \alpha} s(\alpha)\left(T_{\sigma 3 ; \alpha 1}-T_{\sigma 3 ; \alpha 2}\right) / 2 .
$$

The above symmetries imply that $T_{\sigma 1 ; \alpha 4}=T_{\sigma 2 ; \alpha 3}=T_{\bar{\sigma} 3 ; \bar{\alpha} 2}$. This then yields $I_{1}^{s p i n}=-I_{3}^{c}$ which implies the Onsager relation

$$
G_{S C}^{x y}=-G_{C S}^{y x} \text {. }
$$

This is verified numerically in Fig. 6 which presents the disorder averaged spin-Hall conductance and charge Hall conductance for hole systems of different sizes. For a specific disorder realization this relation does not hold and is only approximate depending on the strength of the fluctuations induced by disorder. This relation between $G_{S C}$ and $G_{C S}$ is consistent with predictions of semiclassical wave-packet theory, where standard definition of spin-current was modified by a spin-torque term. ${ }^{28,33}$ However, as seen from the above derivation and noted in the introduction, our finding of an Onsager relations in mesoscopic coherent systems do not involve such spin-torque term since all spin-currents are defined in the non-spin-orbit coupled leads.

\section{SUMMARY}

We have analyzed the spin Hall effect as well as its reciprocal effect in mesoscopic hole and electron systems. We have shown that the spin-Hall as well as the spin-dependent 
chemical potential gradient driven charge-Hall conductances are several times larger for hole systems. Further we have proposed the experimental setup to detect the transverse charge current driven by the spin-dependent chemical potential gradient through transport measurements. We have shown that this charge current is of the order of hundred nano-amperes in hole systems and should be detectable. Also, we have established a direct relation between the disorder average spin-Hall and spin-dependent chemical potential driven charge-Hall conductances in the mesoscopic systems.

\section{ACKNOWLEDGMENTS}

We thank A. H. MacDonald for stimulating discussions. The work was partly supported by the Research Grant Council of Hong Kong under Grant No. HKU 7039/05P (S.Q.S.) and DOE Grant No. DE-FG03-02ER45958 (Q.N.) as well as The Grant Agency of the Czech Republic under Grant No. 202/05/0575 (T.J.).
${ }^{1}$ S. Murakami, N. Nagaosa, and S.-C. Zhang, Science 301, 1348 (2003).

${ }^{2}$ J. Sinova, D. Culcer, Q. Niu, N. A. Sinitsyn, T. Jungwirth, and A. H. MacDonald, Phys. Rev. Lett. 92, 126603 (2004).

${ }^{3}$ M. I. Dyakonov and V. I. Perel, Zh. Eksp. Teor. Fiz. Pis'ma Red. 13, 657 (1971).

${ }^{4}$ J. E. Hirsch, Phys. Rev. Lett. 83, 1834 (1999).

${ }^{5}$ S. Zhang, Phys. Rev. Lett. 85, 393 (2000).

${ }^{6}$ Y. K. Kato, R. C. Myers, A. C. Gossard, and D. D. Awschalom, Science 306, 1910 (2004).

${ }^{7}$ J. Wunderlich, B. Kaestner, J. Sinova, and T. Jungwirth, Phys. Rev. Lett. 94, 047204 (2005).

${ }^{8}$ D. Culcer, J. Sinova, N. A. Sinitsyn, T. Jungwirth, A. H. MacDonald, and Q. Niu, Phys. Rev. Lett. 93, 046602 (2004); N. A. Sinitsyn, E. M. Hankiewicz, W. Teizer, and J. Sinova, Phys. Rev. B 70, 081312(R) (2004); B. A. Bernevig and S.-C. Zhang, condmat/0408442 (unpublished); J. Hu, B. A. Bernevig, and C. Wu, cond-mat/0310093 (unpublished); S. I. Erlingsson, J. Schliemann, and D. Loss, Phys. Rev. B 71, 035319 (2005); S.-Q. Shen, ibid. 70, 081311(R) (2004); S.-Q. Shen, M. Ma, X. C. Xie, and F. C. Zhang, Phys. Rev. Lett. 92, 256603 (2004); L. Hu, J. Gao, and S.-Q. Shen, Phys. Rev. B 69, 165304 (2004); A. A. Burkov, A. S. Nunez, and A. H. MacDonald, ibid. 70, 155308 (2004); S. Zhang and Z. Yang, Phys. Rev. Lett. 94, 066602 (2005).

${ }^{9}$ E. I. Rashba, Phys. Rev. B 68, 241315(R) (2003).

${ }^{10}$ E. I. Rashba, Phys. Rev. B 70, 161201(R) (2004).

${ }^{11}$ O. V. Dimitrova, cond-mat/0405339 (unpublished); cond-mat/ 0407612 (unpublished).

${ }^{12}$ E. G. Mishchenko, A. V. Shytov, and B. I. Halperin, Phys. Rev. Lett. 93, 226602 (2004).

${ }^{13}$ K. Nomura, J. Sinova, T. Jungwirth, Q. Niu, and A. H. MacDonald, Phys. Rev. B 71, 041304(R) (2005).
${ }^{14}$ J. I. Inoue, G. E. W. Bauer, and L. W. Molenkamp, Phys. Rev. B 70, 041303(R) (2004).

${ }^{15}$ O. Chalaev and D. Loss, cond-mat/0407342 (unpublished).

${ }^{16}$ A. Khaetskii, cond-mat/0408136 (unpublished).

${ }^{17}$ B. K. Nikolić, L. P. Zarbo, and Z. Souma, cond-mat/0408693 (unpublished).

${ }^{18}$ L. Sheng, D. N. Sheng, and C. S. Ting, Phys. Rev. Lett. 94, $016602(2005)$

${ }^{19}$ E. M. Hankiewicz, L. W. Molenkamp, T. Jungwirth, and J. Sinova, Phys. Rev. B 70, 241301(R) (2004).

${ }^{20}$ N. Sugimoto, S. Onoda, S. Murakami, and N. Nagaosa, cond-mat/ 0503475 (unpublished).

${ }^{21}$ S. Murakami, cond-mat/0504353 (unpublished).

${ }^{22}$ D. N. Sheng, L. Sheng, Z. Y. Weng, and F. D. M. Haldane, condmat/0504218 (unpublished).

${ }^{23}$ K. Nomura, J. Sinova, N. A. Sinitsyn, and A. H. MacDonald, cond-mat/0506189 (unpublished).

${ }^{24}$ S. Murakami, Phys. Rev. B 69, 241202(R) (2004).

${ }^{25}$ B. A. Bernevig and S.-C. Zhang, cond-mat/0412550 (unpublished).

${ }^{26}$ J. Li, L. Hu, and S.-Q. Shen, cond-mat/0502102 (unpublished).

${ }^{27}$ M. W. Wu and J. Zhou, cond-mat/0503616 (unpublished).

${ }^{28} \mathrm{P}$. Zhang and Q. Niu, cond-mat/0406436 (unpublished).

${ }^{29}$ J. Schliemann and D. Loss, Phys. Rev. B 71, 085308 (2005).

${ }^{30}$ S. Datta, Electronic Transport in Mesoscopic Systems (Cambridge University Press, Cambridge, 1995).

${ }^{31}$ T. Koga, J. Nitta, T. Akazaki, and H. Takayanagi, Phys. Rev. Lett. 89, 046801 (2002).

${ }^{32}$ E. Johnston-Halperin, D. Lofgreen, R. K. Kawakami, D. K. Young, L. Coldren, A. C. Gossard, and D. D. Awschalom, Phys. Rev. B 65, 041306(R) (2002).

${ }^{33}$ P. Zhang, J. Shi, D. Xiao, and Q. Niu, cond-mat/0503505 (unpublished). 